\title{
Papers
}

\section{Observational study of upper gastrointestinal haemorrhage in elderly patients given selective cyclo-oxygenase-2 inhibitors or conventional non-steroidal anti-inflammatory drugs}

\author{
Muhammad Mamdani, Paula A Rochon, David N Juurlink, Alex Kopp, Geoffrey M Anderson, \\ Gary Naglie, Peter C Austin, Andreas Laupacis
}

\begin{abstract}
Objective To compare rates of upper gastrointestinal haemorrhage among elderly patients given selective cyclo-oxygenase-2 (COX 2) inhibitors and non-selective non-steroidal anti-inflammatory drugs (NSAIDs).

Design Observational cohort study.

Setting Administrative data from Ontario, Canada, used from 17 April 2000 to 31 March 2001 to identify population based, NSAID-naive cohorts of patients. Patients Subjects aged $\geqslant 66$ years who started taking non-selective NSAIDs $(\mathrm{n}=5391)$, diclofenac plus misoprostol $(\mathrm{n}=5087)$, rofecoxib $(\mathrm{n}=14583)$, or celecoxib ( $\mathrm{n}=18$ 908) and a randomly selected control cohort not exposed to NSAIDs $(\mathrm{n}=100000)$.

Main outcome measures Rate ratios of hospital admission for upper gastrointestinal haemorrhage in each drug cohort with adjustment for potential confounders.

Results Relative to controls, the multivariate model revealed an increased short term risk of upper gastrointestinal haemorrhage for users of non-selective NSAIDs (adjusted rate ratio 4.0 (95\% confidence intervals 2.3 to 6.9$)$ ), diclofenac plus misoprostol (3.0 (1.7 to 5.6)), and rofecoxib (1.9 (1.3 to 2.8)) but not celecoxib (1.0 (0.7 to 1.6$)$ ). Relative to celecoxib, significantly higher risks of upper gastrointestinal haemorrhage were observed for non-selective NSAIDs (4.4 (2.3 to 8.5)), diclofenac plus misoprostol (3.2 (1.6 to 6.5)), and rofecoxib (1.9 (1.2 to 2.8)). Relative to rofecoxib, non-selective NSAID users were at significantly higher risk of upper

gastrointestinal haemorrhage (1.9 (1.0 to 3.5)).

Conclusions This population based observational study found a lower short term risk of upper gastrointestinal haemorrhage for selective COX-2 inhibitors compared with non-selective NSAIDs.
\end{abstract}

\section{Introduction}

Non-steroidal anti-inflammatory drugs (NSAIDs) are among the most commonly used drugs in the world ${ }^{1}$ and are consumed by about $20-30 \%$ of elderly people in developed countries. ${ }^{23}$ Selective cyclo-oxygenase-2 (COX 2) inhibitors are a new group of NSAIDs that have rapidly gained acceptance in clinical practice. ${ }^{4}$ Within the first three months of its availability, celecoxib became the fastest selling drug in history. ${ }^{5}$

The adoption of selective COX 2 inhibitors has primarily been driven by the assertion that these drugs cause fewer gastrointestinal events than do conventional, non-selective NSAIDs. ${ }^{6}$ Use of conventional NSAIDs is estimated to increase the risk of gastrointestinal complications about fourfold among elderly people, ${ }^{7-10}$ although some drugs may have less gastrotoxic potential than others. ${ }^{11}$ Two large randomised controlled trials that separately evaluated rofecoxib and celecoxib showed that they significantly reduced the number of clinical upper gastrointestinal events compared with conventional NSAIDs. ${ }^{12}{ }^{13}$ Given major differences in study design between these two trials, valid comparisons of the gastrointestinal safety of celecoxib, rofecoxib, and conventional NSAIDs cannot be made from these data alone.

Several important clinical questions remain about the gastrointestinal effects of selective COX 2 inhibitors. It is unclear to what degree they increase gastrointestinal risk relative to not using NSAIDs, and the relative gastrointestinal safety of the different COX 2 inhibitors is uncertain since they have not been directly compared in a single large study. Accordingly, we conducted a population based cohort study to compare the rate of upper gastrointestinal haemorrhage in over 40000 NSAID-naive elderly users of rofecoxib, celecoxib, non-selective NSAIDs, and diclofenac plus misoprostol with that in 100000 nonNSAID users.

\section{Methods}

\section{Study design}

We conducted a population based retrospective cohort study by linking administrative healthcare databases covering over 1.3 million patients aged 66 years or more in Ontario, Canada, from 17 April 2000 through to 31 March 2001. Ontario's elderly population has universal access to prescription drugs, hospital care,
Editorial by Jones See also p 619

Institute for Clinical Evaluative Sciences, 2075 Bayview

Avenue-G215,

Toronto, Ontario,

Canada

Muhammad

Mamdani

scientist

Alex Kopp

analyst

Peter C Austin

scientist

Andreas Laupacis

chief executive officer

Kunin Lunenfeld

Applied Research

Unit, Baycrest

Centre for Geriatric

Care, Toronto

Paula A Rochon

scientist and assistant

director

Sunnybrook and

Women's College

Health Sciences

Centre, Toronto

David N Juurlink clinical

pharmacologist

Department of

health policy,

management, and

evaluation, Faculty

of Medicine,

University of

Toronto

Geoffrey M

Anderson

professor

University of

Toronto

Gary Naglie

Mary Trimmer chair in geriatric medicine research

Correspondence to: M Mamdani muhammad.mamdani@ ices.on.ca

bmj.com 2002;325:624 
and doctor services. This study was approved by the Ethics Review Board of Sunnybrook and Women's College Health Sciences Centre.

\section{Data sources}

The administrative healthcare databases in Ontario allowed for cohort identification, comorbidity assessment, and endpoint ascertainment. The linked databases included computerised pharmacy records of the Ontario Drug Benefit Program, which records prescription drugs dispensed to all Ontario residents aged 65 years or older. Both rofecoxib and celecoxib were first listed on the Ontario Drug Benefit formulary on 17 April 2000 on a limited use basis for patients who did not respond to or were intolerant of traditional NSAIDs or patients with a history of upper gastrointestinal haemorrhage or ulcer. The approved indications for celecoxib included osteoarthritis and rheumatoid arthritis, whereas rofecoxib was approved only for use in osteoarthritis. No such restrictions governed the prescribing of conventional NSAIDs or diclofenac plus misoprostol. We were unable to examine meloxicam use as it was not available on the Ontario Drug Benefit formulary during the study period.

We obtained hospitalisation records from the Canadian Institute for Heath Information Discharge Abstract Database, which contains a detailed record of all hospital admissions, including diagnostic and procedural information. The Ontario Health Insurance Plan provided physician billing information for inpatient and outpatient services, and the Ontario Registered Persons Database contained basic demographic information and vital statistics, including death date, for each Ontario resident. These databases were linked anonymously using encrypted individual health card numbers.

\section{Cohort definition}

We compared users of rofecoxib, celecoxib, nonselective NSAIDs, or the combination of diclofenac plus misoprostol with a random sample of 100000 controls dispensed none of these drugs. Despite the potential differences in morbidity between users of NSAIDs and non-users, we chose patients not using NSAIDs as the control group for two reasons: firstly, such a control group provides useful baseline risk estimates of upper gastrointestinal haemorrhage not related to NSAID use, and, secondly, most previous studies of the association between NSAID use and upper gastrointestinal haemorrhage have non-users of NSAIDs as controls. Thus, choosing non-users of NSAIDs as our control group allowed comparison of our incidence and relative risk estimates with such studies. We also conducted pairwise comparisons of the different NSAID study groups in relation to each other.

For the four drug cohorts, the first NSAID prescription during the study period after a patient's 66 th birthday served as the index date. To create a cohort of NSAID-naive subjects within these four drug groups, we excluded individuals who were dispensed an NSAID in the year preceding the index date. We also excluded subjects given NSAIDs from more than one of the study's four groups of drug on the same day. To exclude sporadic users of NSAIDs, we included only those individuals who were given at least two successive prescriptions of NSAID and who received enough drug for at least 30 days of observation. Events occurring during this initial 30 day period were included in the analysis.

To create the control cohort, all Ontario residents not included in any of the above cohorts were randomly assigned index dates from 17 April 2000 to 15 March 2001, as in the drug cohorts. Individuals aged 66 years and older who were alive on the assigned index date were screened for NSAID use. From those without a prescription for any NSAID in the year before the index date or during the observation period, we randomly selected 100000 individuals to form the control cohort. This group was not matched for age or sex to any of the drug cohorts, but represented the general elderly population of Ontario not prescribed NSAIDs.

We repeated the analyses using controls matched by age (within one year of the birth date) and sex to all patients in the four drug cohorts as a sensitivity analysis. Because women are more likely than men to receive NSAIDs $^{14}$ and may have a lower risk for upper gastrointestinal haemorrhage, ${ }^{7}$ we repeated the analyses separately for men and women. Finally, we repeated the upper gastrointestinal haemorrhage analysis after excluding subjects with a history of such bleeds.

\section{Duration of exposure}

For each of the four drug cohorts, we defined the duration of exposure as the period of continuous, exclusive use of one of the study drug groups starting from the index date. In the non-selective NSAID group, subjects were allowed to switch between different non-selective NSAIDs during the observation period. The "days supply" recorded in the pharmacy claims database allowed us to estimate the intended duration of each prescription. If subjects were given another prescription of the drug before the end of this period, the excess drug supply was carried over to the next prescription's estimated duration. Subjects were allowed a period of grace, $20 \%$ of the period covered by the previous prescription, to obtain another prescription of the drug. If they failed to obtain a repeat prescription within this time, they were deemed to have discontinued the study drug.

Follow up of subjects ended on their admission to hospital for upper gastrointestinal haemorrhage, exposure to an NSAID from another study group, discontinuation of the study drug, death, or the end of the observation period (31 March 2001). We identified hospital admissions for upper gastrointestinal haemorrhage using diagnosis codes ICD-9 (international classification of diseases, ninth revision) $531,532,534$, 578.0, 578.1, and 578.9. Such codes have been shown to yield a positive predictive value of $86 \%$ for upper gastrointestinal haemorrhage. ${ }^{15}$

For the control cohort, each individual was allowed at least 15 days of follow up from the index date, and the end of the observation period was randomly assigned unless a subject was admitted for upper gastrointestinal haemorrhage or died beforehand.

\section{Statistical analysis}

We conducted time-to-event analyses for upper gastrointestinal haemorrhage using Cox proportional hazard models with the control group as the reference. The box lists the covariates used in the model. We 


\section{Covariates assessed in analysis}

\section{Hospitalisations}

Any hospitalisation in preceding year

Malignancy in preceding 5 years

Upper gastrointestinal haemorrhage in preceding 5 years

\section{Procedures}

Gastrointestinal endoscopy or radiological series in preceding 5 years

\section{Drug use}

Number of different drugs in preceding year Narcotic analgesics or gastroprotective agents in preceding 180 days

In the 120 days before index date until end of follow up:

Aspirin

Anticoagulants

Antiplatelets

Antidiabetic agents

Antirheumatics

Glucocorticoids

Gastroprotective agents

\section{Other}

Age

Sex

Long term care

Low income status (annual income of $<\$ C 16018$ (singles) and $<\$$ C24 175 (couples), confirmed through personal tax statements on voluntarily application for reductions in copayments and deductibles)

examined the number of distinct drugs dispensed in the year before the index date as an overall measure of comorbidity ${ }^{16}$ a measure comparable to the Charlson comorbidity index. ${ }^{17}$ We compared all pairwise combinations of hazard ratios for the different drug groups. In each analysis we assessed the proportional hazards assumption for each exposure variable for any violations. All analyses were performed with SAS for UNIX, version 8.2 (SAS Institute, Cary, NC).

\section{Results}

\section{Cohort description}

Of about 1.3 million potential subjects aged 65 years and older, $364686(28 \%)$ were dispensed a prescription NSAID during the study period. From the total elderly population, we identified 5391 users of non-selective NSAIDs, 5087 users of diclofenac plus misoprostol, 14583 users of rofecoxib, 18908 users of celecoxib, and 100000 controls (table 1) who met our inclusion criteria. Among the users of non-selective NSAIDs, most started with naproxen (32\%), ibuprofen $(23 \%)$, or diclofenac $(20 \%)$. A greater proportion of rofecoxib and celecoxib users were women than in the other groups. The control group generally used less healthcare resources than the other study groups. More rofecoxib and celecoxib users had previously undergone upper gastrointestinal diagnostic procedures or received gastroprotective agents than the other groups (table 1). They were also more likely to receive anticoagulants, antirheumatics, and glucocorticoids. The characteristics of the rofecoxib and celecoxib groups, however, were virtually identical.
During over 55000 person years of follow up, we observed 187 hospitalisations for upper gastrointestinal haemorrhage (table 2). Relative to the control group, model based estimates adjusted for the covariates in the box revealed significantly higher risk ratios for users of non-selective NSAIDs (adjusted rate ratio $4.0 ; 95 \%$ confidence interval 2.3 to 6.9$)$, diclofenac plus misoprostol (3.0; 1.7 to 5.6), and rofecoxib (1.9; 1.3 to 2.8 ), but not celecoxib (1.0; 0.7 to 1.6$)$ (see figure). Analyses with age and sex matched controls, separate analyses for men and women, and analyses excluding subjects with a history of upper gastrointestinal haemorrhage all yielded similar findings.

Pairwise comparisons revealed significant differences in risk of upper gastrointestinal haemorrhage among the different drug groups. Relative to celecoxib users, a higher risk of hospitalisation for upper gastrointestinal haemorrhage was seen among users of nonselective NSAIDs (adjusted rate ratio $4.4 ; 2.3$ to 8.5 ), diclofenac plus misoprostol $(3.2 ; 1.6$ to 6.5$)$, and rofecoxib $(1.9 ; 1.2$ to 2.8$)$. Relative to rofecoxib, a significantly higher risk of upper gastrointestinal haemorrhage was observed for non-selective NSAIDs (1.9; 1.0 to 3.5$)$ but not diclofenac plus misoprostol $(1.4 ; 0.7$ to 2.7$)$.

We conducted several sensitivity analyses. Firstly, we repeated the analysis examining all individuals who were given an NSAID irrespective of the number of prescriptions dispensed or the quantity of drug supplied. The findings were similar to those of the primary analysis. Secondly, we limited our analysis to patients not residing in a long term care institution and also found results similar to those of the primary analysis. Thirdly, we repeated the analysis among users of gastroprotective agents and non-users given the substantial discrepancy in use of gastroprotective agents observed in the various cohorts. Subjects given gastroprotective agents had a higher incidence of upper gastrointestinal haemorrhage than subjects in their respective groups not given gastroprotective agents during follow up, implying that these agents are

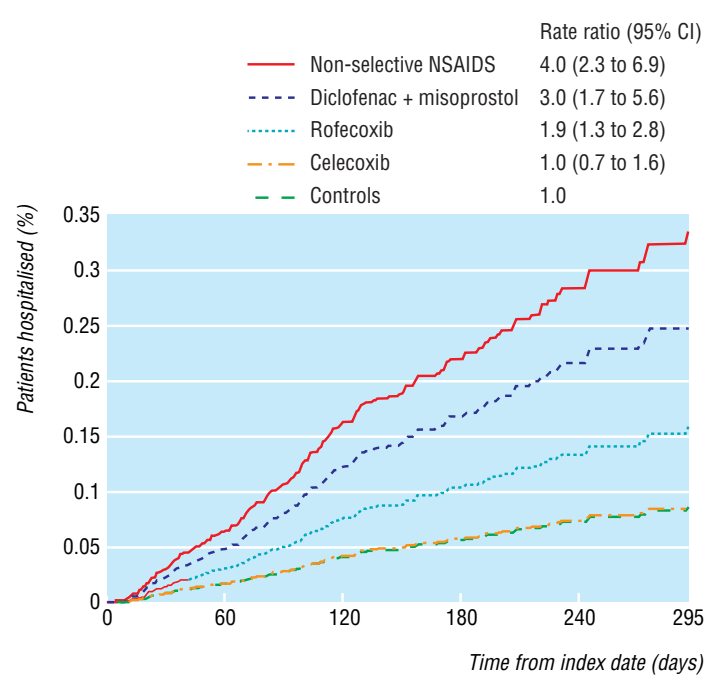

Adjusted Cox proportional hazard estimates for hospitalisation for upper gastrointestinal haemorrhage among elderly patients using prescribed NSAIDs 
Table 1 Characteristics of cohorts in study of elderly patients using different NSAIDs. Values are numbers (percentages) unless stated otherwise

\begin{tabular}{|c|c|c|c|c|c|}
\hline & \multicolumn{5}{|c|}{ Study cohort } \\
\hline & $\begin{array}{l}\text { Community } \\
\text { controls }\end{array}$ & $\begin{array}{l}\text { Non-selective } \\
\text { NSAIDs }\end{array}$ & $\begin{array}{c}\text { Diclofenac }+ \\
\text { misoprostol }\end{array}$ & Rofecoxib & Celecoxib \\
\hline No of patients (\% women) & $100000(55)$ & $5391(59)$ & $5087(62)$ & $14583(72)$ & $18908(70)$ \\
\hline Mean (SD) age (years) & $75.4(7.3)$ & $75.5(7.0)$ & $76.6(7.1)$ & $76.5(6.9)$ & $76.5(6.8)$ \\
\hline Residence in long term care facility & $4074(4)$ & $398(7)$ & $503(10)$ & $652(4)$ & $810(4)$ \\
\hline Low income status & $21073(21)$ & $1831(34)$ & $1725(34)$ & $4445(30)$ & $5673(30)$ \\
\hline Hospitalisation in past year & $11513(12)$ & $1023(19)$ & $925(18)$ & $2900(20)$ & 3651 (19) \\
\hline Mean (SD) No of prescription drugs in past year & $5.4(5.4)$ & $8.3(6.4)$ & $8.3(6.4)$ & $9.9(6.5)$ & $9.5(6.4)$ \\
\hline $\begin{array}{l}\text { Use of gastroprotective agents within } 180 \text { days before } \\
\text { entry to cohort }\end{array}$ & 17279 (17) & $1329(25)$ & $1265(25)$ & $6140(42)$ & 7738 (41) \\
\hline $\begin{array}{l}\text { Use of narcotic analgesics within } 180 \text { days before entry to } \\
\text { cohort }\end{array}$ & $10623(11)$ & $1419(26)$ & $1321(26)$ & $4511(31)$ & $5587(30)$ \\
\hline \multicolumn{6}{|l|}{ Hospitalisations or procedures in past 5 years: } \\
\hline Malignancy & $4785(5)$ & $371(7)$ & $294(6)$ & $760(5)$ & $1004(5)$ \\
\hline Prior upper gastrointestinal haemorrhage & $1440(1)$ & $64(1)$ & $66(1)$ & $369(3)$ & $476(3)$ \\
\hline Prior gastrointestinal or radiological procedure & $17839(18)$ & $1090(20)$ & $1043(21)$ & 4731 (32) & 5855 (31) \\
\hline \multicolumn{6}{|l|}{ Drug use in 120 days before index date to end of follow up: } \\
\hline Aspirin & 11564 (12) & $1014(19)$ & $945(19)$ & $2629(18)$ & 3311 (18) \\
\hline Anticoagulants & $6716(7)$ & $244(5)$ & $266(5)$ & $1515(10)$ & $1929(10)$ \\
\hline Antihyperglycaemics & $9256(9)$ & 756 (14) & 706 (14) & $1819(12)$ & $2344(12)$ \\
\hline Antirheumatics & 0 & $66(1)$ & $71(1)$ & $401(3)$ & $865(5)$ \\
\hline Glucocorticoids & 3789 (4) & $458(9)$ & $384(8)$ & $1928(13)$ & 2471 (13) \\
\hline Gastroprotective agents: & $16394(16)$ & $1699(32)$ & $1277(25)$ & $6213(43)$ & $7793(41)$ \\
\hline Proton pump inhibitors & $6139(6)$ & $432(8)$ & $405(8)$ & $3156(22)$ & $3868(20)$ \\
\hline Other* & 11615 (12) & $1407(26)$ & $983(19)$ & $3754(26)$ & $4778(25)$ \\
\hline
\end{tabular}

*Includes histamine- $\mathrm{H}_{2}$ receptor antagonists, misoprostol, and sucralfate.

selectively prescribed to those at higher risk of upper gastrointestinal haemorrhage. Differences in the risk of upper gastrointestinal haemorrhage between users of non-selective NSAIDs and of the COX 2 inhibitors were present whether or not patients received gastroprotective agents. Fourthly, we examined the doses used in the celecoxib and rofecoxib groups at the time of the last observed prescription, since some evidence indicates that other selective COX 2 drugs may lose their selectivity at higher doses. ${ }^{18}$ Both rofecoxib and celecoxib are approved for osteoarthritis, presumably the most prevalent indication in this cohort, for which $25 \mathrm{mg}$ of rofecoxib and $200 \mathrm{mg}$ of celecoxib are considered to be at the upper end of the dose ranges. ${ }^{19}$ A significantly greater proportion of patients given celecoxib (19\%) were given high doses ( $>200 \mathrm{mg}$ daily) compared with patients given rofecoxib ( $8 \%$ given $>25 \mathrm{mg}$ daily).

\section{Discussion}

Our findings suggest a lower risk of upper gastrointestinal haemorrhage associated with use of selective
COX 2 inhibitors than with conventional, non-selective NSAIDs. The rate of haemorrhage with celecoxib was similar to that among the control group not using NSAIDs. While the risk of haemorrhage with rofecoxib was significantly lower than that with non-selective NSAIDs, it was significantly higher than that with celecoxib.

\section{Study limitations}

Although we attempted to control for many important confounders, we were unable to account for some potentially important factors such as smoking and alcohol consumption. The distribution of such factors among the different groups studied and the consequent influences on our findings is unknown. However, despite a potentially heavier disease burden among the patients using rofecoxib and celecoxib (as a result of the limited use of selective COX 2 inhibitors licensed in Ontario), they had lower risk ratios than the patients using non-selective NSAIDs. Our population based incidence estimates for upper gastrointestinal haemorrhage (table 2) among the controls and non-selective NSAID group are also consistent with those of other

Table 2 Upper gastrointestinal haemorrhage among elderly patients using different NSAIDs

\begin{tabular}{|c|c|c|c|c|c|}
\hline & \multicolumn{5}{|c|}{ Study cohort } \\
\hline & $\begin{array}{l}\text { Community controls } \\
(\mathrm{n}=\mathbf{1 0 0} \mathbf{0 0 0})\end{array}$ & $\begin{array}{l}\text { Non-selective } \\
\text { NSAIDs }(n=5391)\end{array}$ & $\begin{array}{c}\text { Diclofenac + misoprostol } \\
(\mathrm{n}=5087)\end{array}$ & $\begin{array}{l}\text { Rofecoxib } \\
(\mathrm{n}=14583)\end{array}$ & $\begin{array}{l}\text { Celecoxib } \\
(\mathrm{n}=18 \text { 908) }\end{array}$ \\
\hline $\begin{array}{l}\text { No of admissions for upper gastrointestinal } \\
\text { haemorrhage }\end{array}$ & 82 & 17 & 13 & 43 & 32 \\
\hline Mean (SD) days of follow up & $138.7(77.4)$ & $91.7(68.3)$ & $97.8(71.2)$ & $146.9(89.6)$ & $170.3(97.0)$ \\
\hline Total follow up (person years) & 37981 & 1353 & 1361 & 5865 & 8818 \\
\hline $\begin{array}{l}\text { No of upper gastrointestinal haemorrhages } \\
\text { per } 1000 \text { person years }\end{array}$ & 2.2 & 12.6 & 9.6 & 7.3 & 3.6 \\
\hline \multicolumn{6}{|l|}{ Model based risk ratios $(95 \% \mathrm{Cl})$} \\
\hline Unadjusted & 1.0 (reference) & $6.1(3.6$ to 10.2$)$ & 4.6 (2.5 to 8.2$)$ & $3.5(2.4$ to 5.0$)$ & 1.7 (1.1 to 2.6$)$ \\
\hline Adjusted & 1.0 (reference) & $4.0(2.3$ to 6.9$)$ & $3.0(1.7$ to 5.5$)$ & $1.9(1.3$ to 2.8$)$ & $1.0(0.7$ to 1.6$)$ \\
\hline Number needed to treat to harm (NNT(H))* & N/A & 403 & 592 & 1389 & N/A \\
\hline
\end{tabular}

${ }^{*}$ NNT $(H)$ calculations are based on a follow up of 295 days from the Cox proportional hazard model estimates. 
studies, ${ }^{80}$ as are our relative risks. ${ }^{7-10}$ In addition, when we analysed results among both users and non-users of gastroprotective agents, we still found lower adjusted relative risks for upper gastrointestinal haemorrhage for users of selective COX 2 inhibitors than among non-selective NSAID users. Users of gastroprotective agents had a higher incidence of upper gastrointestinal haemorrhage than subjects in their respective groups not dispensed gastroprotective agents. This implies that these agents were selectively prescribed to those at higher risk of upper gastrointestinal haemorrhage and were a marker for underlying gastrointestinal disease associated with a higher risk of upper gastrointestinal haemorrhage.

A second limitation is that we used administrative databases to identify and define exposure to study drugs and clinical outcomes. We have no direct measure of adherence or appropriateness of use. Since NSAIDs may be used in varying doses over time for symptom control, dose equivalence of the various drugs could not be adequately examined with these data. Instead, the NSAIDs were examined as they are commonly used in this population.

We were unable to identify use of non-prescription NSAIDs. However, ibuprofen and aspirin are the only non-prescription non-selective NSAIDs available in Canada, and subjects in our study have a strong financial incentive to obtain these drugs by prescription, especially with regular use. Over a quarter of the elderly subjects were given a NSAID during the observation period, consistent with previous studies examining the use of prescription and non-prescription NSAIDs among elderly people. ${ }^{23}$ This implies that the vast majority of NSAID use in our population is probably captured by our databases. The use of nonprescription aspirin is perhaps the biggest problem, but since the distribution of prescription aspirin use was similar in the study drug groups, the use of non-prescription aspirin is also likely to be equally distributed.

We identified outcomes using diagnostic codes that have been validated previously, but we were unable to capture other important information such as the severity of the gastrointestinal haemorrhage and more subtle outcomes such as non-bleeding ulcers. Also, it is possible that upper gastrointestinal haemorrhage is more readily diagnosed or reported among users of traditional NSAIDs than among users of specific COX 2 inhibitors. However, the diagnosis is not generally difficult to make, its coding has been validated, and the impact of this potential bias is likely minimal.

The low absolute number of events in the study groups precluded reliable subgroup analyses such as comparisons among users of anticoagulants or individual NSAIDs, and the generalisability of our findings to younger patients or settings with different drug policies over longer periods of follow up is uncertain.

\section{Relative gastrointestinal safety of rofecoxib and celecoxib}

Currently, comparisons between rofecoxib and celecoxib are based largely on data from clinical trials and studies of whole blood assays. Two large randomised trials separately comparing rofecoxib ${ }^{12}$ and celecoxib ${ }^{13}$ with non-selective NSAIDs provided similar relative risk reductions of $40-60 \%$ in the incidence of clinical upper gastrointestinal haemorrhage events (that is, upper gastrointestinal haemorrhage ulcer complications plus symptomatic ulcers). However, valid comparisons of upper gastrointestinal haemorrhage event rates between rofecoxib and celecoxib cannot be made from such data for several reasons. Firstly, in the absence of a direct comparison, conclusions about the relative gastrointestinal safety of these drugs are largely speculative. Secondly, the primary endpoints of the two trials were slightly different. Thirdly, the comparator groups, using nonselective NSAIDs, in the two trials were different: the non-selective NSAIDs assessed in the celecoxib trial were either ibuprofen or diclofenac, whereas naproxen was assessed in the rofecoxib study. Since naproxen is probably more gastrotoxic than ibuprofen or diclofenac, ${ }^{11}$ it is difficult to assess the relative gastrointestinal safety of rofecoxib and celecoxib from these two trials. Fourthly, the interpretation of the celecoxib trial is complicated by the nature of its reporting. ${ }^{21}$ The findings were based on a combined analysis of the first six months of two separate and longer trials whose protocols differed substantially from the published paper in design, outcomes, duration of follow up, and analysis. Although 12 month data revealed no significant differences between celecoxib and its non-selective NSAID comparators with respect to complicated ulcer outcomes (the primary endpoint of the trials), the incidence of clinical upper gastrointestinal haemorrhage events remained significantly different. ${ }^{22}$

Our understanding of the cellular effects of the COX 2 inhibitors is also evolving, and conclusions about the relative safety of these agents based on in vitro data may be premature. For example, although whole blood assay studies suggest that rofecoxib is more COX 2 selective than celecoxib, ${ }^{23}$ such assays have been criticised for having limited clinical relevance. ${ }^{24}$ Furthermore, recent studies of cancer cell lines have shown what may be COX independent differences in antiproliferative activity between celecoxib and rofecoxib. ${ }^{25-27}$ The clinical implications of such differences for the gastrointestinal safety of these two drugs are not known.

Our evaluation represents the first direct comparison of rofecoxib and celecoxib for a clinically meaningful gastrointestinal outcome using common comparator groups over the same period, with data reflecting clinical practice. The demographic characteristics of rofecoxib and celecoxib users were strikingly similar in our study, implying that selection of one COX 2 inhibitor over another is probably arbitrary in clinical practice. Therefore, the differences in unobserved covariates between the rofecoxib and celecoxib groups are probably minimal and would not explain the difference in upper gastrointestinal haemorrhage observed between the two drugs.

\section{Conclusions}

Our study found lower rates of upper gastrointestinal haemorrhage with selective COX 2 inhibitors than with non-selective NSAIDs. The significantly higher rate of upper gastrointestinal haemorrhage among users of rofecoxib than users of celecoxib was unexpected. Although the absolute difference in rates 


\section{What is already known on this topic}

Long term NSAID use is associated with the development of peptic and duodenal ulcers

Selective COX 2 inhibitors are claimed to cause fewer gastrointestinal problems than conventional, non-selective NSAIDs

It is unclear to what degree COX 2 inhibitors increase gastrointestinal risk relative to not using NSAIDs, and the relative gastrointestinal safety of the different COX 2 inhibitors is uncertain

\section{What this study adds}

The risk of upper gastrointestinal haemorrhage with the COX 2 inhibitors rofecoxib and celecoxib was significantly lower than with conventional NSAIDs, but the risk with rofecoxib was significantly higher than that with celecoxib

The risk of gastrointestinal haemorrhage with celecoxib was similar to that in controls not using NSAIDs

of upper gastrointestinal haemorrhage was small, the difference, if true, is clinically important given the large numbers of patients prescribed selective COX 2 inhibitors. Large randomised controlled trials directly comparing the drugs are urgently needed to better examine these differences.

Contributors: MM, PAR, DNJ, GMA, GN, and AL designed the study; MM, DNJ, PCA, and AK performed the study. GN, PCA, and AL advised and supervised. Statistical advice was given by PCA. MM is the guarantor.

Funding: MM is supported by a New Investigator award from the New Emerging Teams (NET) of the Canadian Institutes of Health Research (CIHR). PAR is supported by a Career Scientist award from the CIHR. DNJ is supported by a fellowship award from the CIHR and from the ClinicianScientist Program of the Department of Medicine at the University of Toronto. AL is a senior scientist of the CIHR. This study was supported by a CIHR operating grant (MOP-49527) and a CIHR Chronic Disease New Emerging Team program grant (NET-54010). The NET program receives joint sponsorship from the Canadian Diabetes Association, the Kidney Foundation of Canada, the Heart and Stroke Foundation of Canada, and the CIHR Institutes of Nutrition, Metabolism, and Diabetes and Circulatory and Respiratory Health.

Competing interests: MM has conducted research in an unrelated content area at the request of an academic institution whose funding was supported by Pharmacia in the past two years, but none of the funding for this study was provided by any pharmaceutical company.

Misoprostol for co-prescription with NSAIDs. Drug Ther Bull 1990;28:25-6

2 Barat I, Andreasen F, Damsgaard EMS. The consumption of drugs by 75-year-old individuals living in their own homes. Eur J Clin Pharmacol 2000;56:501-9.
3 Sayer GP, Britt H, Horn F, Bhasale A, McGeechan K, Charles J, et al Measures of health and health care delivery in general practice in Australia. Australian Institute of Health and Welfare. April 2000. (www.aihw.gov.au publications/health/mhhcdgpa/index.html (accessed 27 May 2002))

4 Hawkey CJ. COX-2 inhibitors. Lancet 1999:353:307-14.

5 IMS Health Canada. New arthritis medication achieves fastest adoption ever recorded in Canada. Newsletter September 1999 (www.imshealthcanada.com/htmen/4_2_1_14.htm)

6 Feldman M, McMahon AT. Do cyclooxygenase-2 inhibitors provide benefits similar to those of traditional nonsteroidal anti-inflammatory drugs, with less gastrointestinal toxicity? Ann Intern Med 2000;132:134-43.

7 Hernandez-Diaz S, Rodriguez LA. Association between nonsteroidal anti-inflammatory drugs and upper gastrointestinal tract bleeding/ perforation: an overview of epidemiologic studies published in the 1990s. Arch Intern Med 2000;160:2093-9.

8 Aalykke C, Lauritsen K. Epidemiology of NSAID-related gastroduodenal mucosal injury. Best Pract Res Clin Gastroenterol 2001;15:705-22.

9 Griffin MR, Piper JM, Daugherty JR, Snowden M, Ray WA. Nonsteroidal anti-inflammatory drug use and increased risk for peptic ulcer disease in elderly persons. Ann Intern Med 1991;114:257-63.

10 Gabriel SE, Jaakkimainen L, Bombardier C. Risk for serious gastrointestinal complications related to use of nonsteroidal antiinflammatory drugs. A meta-analysis. Ann Intern Med 1991;115:787-96.

11 Henry D, Lim LL, Garcia Rodriguez LA, Perez Gutthann S, Carson JL, Griffin M, et al. Variability in risk of gastrointestinal complications with individual non-steroidal anti-inflammatory drugs: results of a collaborative meta-analysis. $B M J$ 1996;312:1563-6.

12 Bombardier C, Laine L, Reicin A, Shapiro D, Burgos-Vargas R, Davis B, et al. Comparison of upper gastrointestinal toxicity of rofecoxib and naproxen in patients with rheumatoid arthritis. $N$ Engl $J$ Med 2000;343:1520-8.

13 Silverstein FE, Faich G, Goldstein JL, Simon LS, Pincus T, Whelton A, et al. Gastrointestinal toxicity with celecoxib vs nonsteroidal antiinflammatory drugs for osteoarthritis and rheumatoid arthritis: the CLASS study: a randomized controlled trial. JAMA 2000;284:1247-55.

14 Lassila HC, Stoehr GP, Ganguli M, Seaberg EC, Gilby JE, Belle SH, et al Use of prescription medications in an elderly rural population: the MoVIES Project. Ann Pharmacother 1996;30:589-95.

15 Raiford DS, Gutthann SP, Rodriguez LAG. Positive predictive value of ICD.9 codes in the identification of cases of complicated peptic ulcer disease in the Saskatchewan hospital automated database. Epidemiology 1996; $7: 101.4$

16 Schneeweiss S, Seeger JD, Maclure M, Wang PS, Avorn J, Glynn RJ Performance of comorbidity scores to control for confounding in epidemiologic studies using claims data. Am J Epidemiol 2001;154:854-64.

17 Charlson ME, Pompei P, Ales KL, MacKenzie CR. A new method of classifying prognostic comorbidity in longitudinal studies: Development and validation. J Chron Dis 1987;40:373-83.

18 Patrignani P, Panara MR, Sciulli MG, Santini G, Renda G, Patrono C. Differential inhibition of human prostaglandin endoperoxide synthase-1 and -2 by nonsteroidal anti-inflammatory drugs. I Physiol Pharmacol 1997;48:623-31

19 Fitzgerald GA, Patrono C. The coxibs, selective inhibitors of cyclooxygenase-2. N Engl J Med 2001;345:433-42.

20 Hernandez-Diaz S, Rodriguez LAG. Incidence of serious upper gastrointestinal bleeding/perforation in the general population: review of epidemiologic studies. J Clin Epidemiol 2002;55:157-63.

21 Juni P, Rutjes AWS, Dieppe PA. Are the COX 2 inhibitors superior to traditional non-steroidal anti-inflammatory drugs? BMJ 2002;324:1287-8.

$22 \mathrm{Lu} \mathrm{HL}$. Statistical reviewer briefing document for the advisory committee www.fda.gov/ohrms/dockets/ac/01/briefing/3677b1_04_stats.doc (accessed 3 June 2002)

23 Warner TD, Giuliano F, Vojnovic I, Bukasa A, Mitchell JA, Vane JR. Nonsteroid drug selectivities for cyclo-oxygenase-1 rather than cyclooxygenase-2 are associated with human gastrointestinal toxicity: a full in vitro analysis. Proc Natl Acad Sci U S A 1999:96:7563-8.

24 Blain H, Boileau C, Lapicque F, Nedelec E, Loeuille D, Guillaume C, et al. Limitation of the in vitro whole blood assay for predicting the COX selectivity of NSAIDs in clinical use. Br J Clin Pharmacol 2002;53:255-65.

25 Hawk ET, Viner JL, Dannenberg A, Dubois RN. COX-2 in cancer-a player that's defining the rules. J Natl Cancer Inst 2002;94:545-6.

26 Patti R, Gumired K, Reddanna P, Sutton LN, Phillips PC, Reddy CD. Overexpression of cyclooxygenase-2 (COX-2) in human primitive neuroectodermal tumors: effect of celecoxib and rofecoxib. Cancer Lett 2002;180(1):13-21.

27 Waskewich C, Blumenthal RD, Li H, Stein R, Goldenberg DM, Burton J Celecoxib exhibits the greatest potency amongst cyclooxygenase (COX) inhibitors for growth inhibition of COX-2-negative hematopoietic and epithelial cell lines. Cancer Res 2002;62:2029-33.

(Accepted 12 August 2002) 